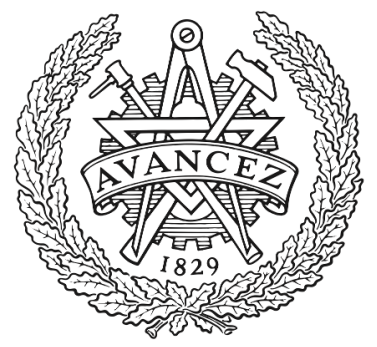

CHALMERS

UNIVERSITY OF TECHNOLOGY

\title{
First Direct Measurement of Isotopic Fission-Fragment Yields of 239U
}

Downloaded from: https://research.chalmers.se, 2023-04-26 11:54 UTC

Citation for the original published paper (version of record):

Ramos, D., Caamano, M., Lemasson, A. et al (2019). First Direct Measurement of Isotopic Fission-Fragment Yields of 239U. Physical Review Letters, 123(9).

http://dx.doi.org/10.1103/PhysRevLett.123.092503

N.B. When citing this work, cite the original published paper. 


\title{
First Direct Measurement of Isotopic Fission-Fragment Yields of ${ }^{239} \mathbf{U}$
}

\author{
D. Ramos, ${ }^{1,2,{ }^{*}}$ M. Caamaño, ${ }^{3}$ A. Lemasson, ${ }^{2}$ M. Rejmund,${ }^{2}$ L. Audouin, ${ }^{1}$ H. Álvarez-Pol, ${ }^{3}$ J. D. Frankland, ${ }^{2}$ \\ B. Fernández-Domínguez, ${ }^{3}$ E. Galiana-Baldó, ${ }^{3,4}$ J. Piot, ${ }^{2}$ D. Ackermann, ${ }^{2}$ S. Biswas, ${ }^{2}$ E. Clement, ${ }^{2}$ D. Durand, ${ }^{5}$ \\ F. Farget, ${ }^{5}$ M. O. Fregeau, ${ }^{2}$ D. Galaviz, ${ }^{4}$ A. Heinz, ${ }^{6}$ A. I. Henriques, ${ }^{7}$ B. Jacquot, ${ }^{2}$ B. Jurado, ${ }^{7}$ Y. H. Kim, ${ }^{2, \dagger}$ P. Morfouace, ${ }^{2, \$}$ \\ D. Ralet, ${ }^{8}$ T. Roger, ${ }^{2}$ C. Schmitt, ${ }^{9}$ P. Teubig, ${ }^{4}$ and I. Tsekhanovich ${ }^{7}$ \\ ${ }^{1}$ IPN Orsay, Université de Paris-Saclay, CNRS/IN2P3, F-91406 Orsay Cedex, France \\ ${ }^{2}$ GANIL, CEA/DRF-CNRS/IN2P3, BP 55027, F-14076 Caen Cedex 5, France \\ ${ }^{3}$ IGFAE, Universidade de Santiago de Compostela, E-15706 Santiago de Compostela, Spain \\ ${ }^{4}$ LIP Lisboa, 1649-003 Lisbon, Portugal \\ ${ }^{5}$ LPC Caen, Université de Caen Basse-Normandie-ENSICAEN-CNRS/IN2P3, F-14050 Caen Cedex, France \\ ${ }^{6}$ Chalmers University of Technology, SE-41296 Göteborg, Sweden \\ ${ }^{7}$ CENBG, IN2P3/CNRS-Université de Bordeaux, F-33175 Gradignan Cedex, France \\ ${ }^{8}$ CSNSM, CNRS/IN2P3, Université de Paris-Saclay, F-91405 Orsay, France \\ ${ }^{9}$ IPHC Strasbourg, Université de Strasbourg-CNRS/IN2P3, F-67037 Strasbourg Cedex 2, France
}

(Received 6 June 2019; published 30 August 2019)

\begin{abstract}
A direct and complete measurement of isotopic fission-fragment yields of ${ }^{239} \mathrm{U}$ has been performed for the first time. The ${ }^{239} \mathrm{U}$ fissioning system was produced with an average excitation energy of $8.3 \mathrm{MeV}$ in one-neutron transfer reactions between a ${ }^{238} \mathrm{U}$ beam and a ${ }^{9} \mathrm{Be}$ target at Coulomb barrier energies. The fission fragments were detected and isotopically identified using the VAMOS++ spectrometer at the GANIL facility. The measurement allows us to directly evaluate the fission models at excitation energies of fast neutrons, which are relevant for next-generation nuclear reactors. The present data, in agreement with model calculations, do not support the recently reported anomaly in the fission-fragment yields of ${ }^{239} \mathrm{U}$, and they confirm the persistence of spherical shell effects in the Sn region at excitation energies exceeding the fission barrier by a few mega-electron volts.
\end{abstract}

DOI: 10.1103/PhysRevLett.123.092503

Eighty years after its discovery $[1,2]$, fission continues to play a major role in the production of electricity [3]; and it is a key process for the management and the transmutation of long-lived radioactive nuclear waste [4]. Fissioning systems also serve as natural laboratories to study nuclear dynamics [5-8], are tools to produce neutron-rich nuclei and study their structure [5,9], and play a role in the $r$-process nucleosynthesis [10]. However, a complete microscopic quantum description of the fission process is still lacking [11]. At low excitation energy, the fission mechanism is particularly challenging because of the complex interplay of dynamic and static properties that drives the fissioning system to fission fragments $[12,13]$. This includes nuclear configurations far from equilibrium, the interplay of collective and intrinsic degrees of freedom, and the dynamics of large amplitude collective motion [14-18].

The description of the fission process strongly relies on available experimental information obtained from the final fission fragments [13]. The key observables are fission yields, kinetic energies, and deexcitation schemes of fission fragments. Until recently, the access to these observables was limited to neutron-induced fission on long-lived or stable nuclei. The complete fission-fragment identification was not feasible due to the low kinetic energy of the fission products. The use of surrogate reactions gave access to the study of a wider range of compound nuclei, which were otherwise inaccessible [19-21]. The use of inverse kinematics allowed the direct measurement of the atomic number of complete fission-fragment distributions [22]. In the last decade, the simultaneous use of surrogate reactions, inverse kinematics, and magnetic spectrometers has opened a new field of study measuring complete isotopic fission-fragment distributions [23-26] and leading to an improved understanding of the fission process [12].

There is a need for accurate experimental information on fission fragments because the state-of-art evaluated data for many systems at fast-neutron energies [27,28] rely on interpolations and empirical models. This is required, in particular, for the modeling of next-generation reactors, such as future fast reactors and accelerator-driven systems [29]. The above-mentioned surrogate reactions at Coulomb barrier energies involving transfer-induced fission $[23,26]$ represent unique opportunities to collect such relevant data.

In this Letter, the first direct measurement of the isotopic fission-fragment yields of ${ }^{239} \mathrm{U}$ is reported. These results confirm the persistence of the fission path characterized by a heavy fragment at low deformation (standard I mode [17]) at fast-neutron energies. Recently, anomalies in the 
fission-fragment yields were reported for neutron-induced fission of ${ }^{238} \mathrm{U}$ [30], with large deviations (up to 600\%) with respect to models. These anomalies would change the evaluation of the heat from fission-fragment $\gamma$ decays, which is necessary for modeling present and future reactors. For instance, ${ }^{238} \mathrm{U}(n, f)$ reactions contribute with 2 to $5 \%$ of the total fission rate in current pressurized water reactors [31,32], and they compete with neutron-capture reactions in certain future fast-reactor designs. The present work rules out the reported anomalies on the fissionfragment yields of Mo and $\mathrm{Sn}$, and it gives reliable constraints for current fission models at fast-neutron energies.

The experiment was performed at GANIL using a beam of ${ }^{238} \mathrm{U}$ at $5.88 \mathrm{MeV} /$ nucleon impinging on a $500-\mu \mathrm{g} / \mathrm{cm}^{2}-$ thick ${ }^{9} \mathrm{Be}$ target. ${ }^{239} \mathrm{U}$ was produced in flight in one-neutron transfer reactions $\left[{ }^{9} \mathrm{Be}\left({ }^{238} \mathrm{U},{ }^{239} \mathrm{U}\right){ }^{8} \mathrm{Be}\right]$ with a range of excitation energies high enough to overcome the fission barrier $\left(B_{f}=6.4 \mathrm{MeV}\right.$ [33]) and undergo fission. The fission fragments were detected in the VAMOS++ magnetic spectrometer [34] in coincidence with two $\alpha$ particles resulting from the breakup of ${ }^{8} \mathrm{Be}$, which were detected in the SPIDER telescope [35] and placed $31 \mathrm{~mm}$ downstream from the target. The segmentation of SPIDER provides a measurement of the angle of recoil from 35 to $55^{\circ}$ with respect to the beam axis. This, combined with the measurement of the total energy, allows an event-byevent determination of the total excitation energy of the system with a typical resolution of $1.7 \mathrm{MeV}$. Due to the kinematical focussing, fission fragments were emitted at forward angles within a cone of $\sim 30^{\circ}$. For each measured fission event, one of the two fragments was fully characterized in terms of the mass number, the atomic number, the atomic charge, and the velocity vector using the VAMOS++ spectrometer and its associated detectors. The VAMOS++ spectrometer was rotated by 14 and $21.5^{\circ}$ with respect to the beam axis to optimize the acceptance of heavy and light fragments, respectively. Further details on VAMOS++ along with typical performances for the fission-fragment detection were given in Refs. [34,36,37].

Figure 1(a) shows the identification spectrum of targetlike ions, detected in coincidence with fission fragments, obtained from the correlation of the energy loss and the total energy, which was measured in SPIDER. The energy loss of the two coincident $\alpha$ particles, detected in the same sector, is similar to the energy loss of ${ }^{7} \mathrm{Li}$. Therefore, the selection of actual $\alpha-\alpha$ coincidences is obtained by selecting events where each $\alpha$ particle hits a different ring within the same sector. For such events, the energy loss in the sector is twice the energy loss in each ring. This is shown in Fig. 1(b), where the $\alpha-\alpha$ coincidence was not applied. At the edge of the detector, one $\alpha$ particle may escape from the telescope without hitting the second detector. In such a case, the measurement of the energy
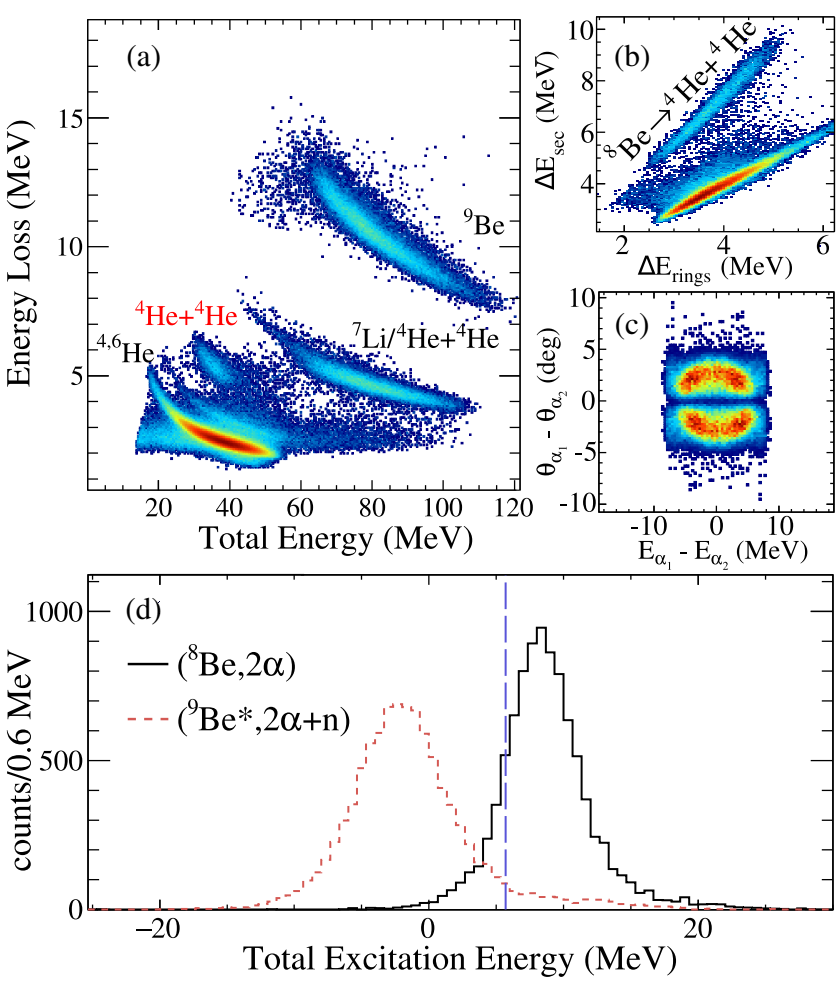

FIG. 1. Identification of targetlike ions detected in coincidence with fission fragments and total excitation energy of the fissioning system: (a) Correlation between energy loss and total energy for targetlike ions detected in SPIDER telescope. (b) Correlation of energy loss measured in rings and the corresponding sector. (c) Correlation between the polar angle and the energy difference of two coincident $\alpha$ particles. (d) Reconstructed total excitation energy of fissioning system, in coincidence with fission events, assuming either breakup of ${ }^{8} \mathrm{Be}$ (solid line) or ${ }^{9} \mathrm{Be}$ (dotted line). Vertical dashed line represents fission barrier of ${ }^{238} \mathrm{U}$ (see text for details).

is incomplete, and those events were discarded [red label in Fig. 1(a)]. Figure 1(c) shows the correlation between the polar angle and the energy difference of coincident $\alpha$ particles that correspond to two-body decay. In Fig. 1(d), the reconstructed total excitation energy distribution, obtained by assuming that the two coincident $\alpha$ particles follow the breakup of ${ }^{8} \mathrm{Be}$, is shown with a solid line for those events detected in coincidence with fission fragments.

These events can contain a small fraction corresponding to the breakup of ${ }^{9} \mathrm{Be}$ from its unbound first excited state at $1.684 \mathrm{MeV}$ [38]. The dotted line of Fig. 1(d) shows the distribution assuming the breakup of ${ }^{9} \mathrm{Be}$. It can be seen that, in the case of ${ }^{9} \mathrm{Be}$ breakup, only the small fraction of the excitation energy distribution above the fission barrier of ${ }^{238} \mathrm{U}$ ( $B_{f}=5.7 \mathrm{MeV}$ [33]), which is indicated by the vertical dashed line, can undergo fission. These events, with excitation energies above $15 \mathrm{MeV}$ and assuming ${ }^{8} \mathrm{Be}$ breakup, are not considered in the following analysis to avoid contamination. Additional contamination from random coincidences between the ${ }^{8} \mathrm{Be}$ and fission 


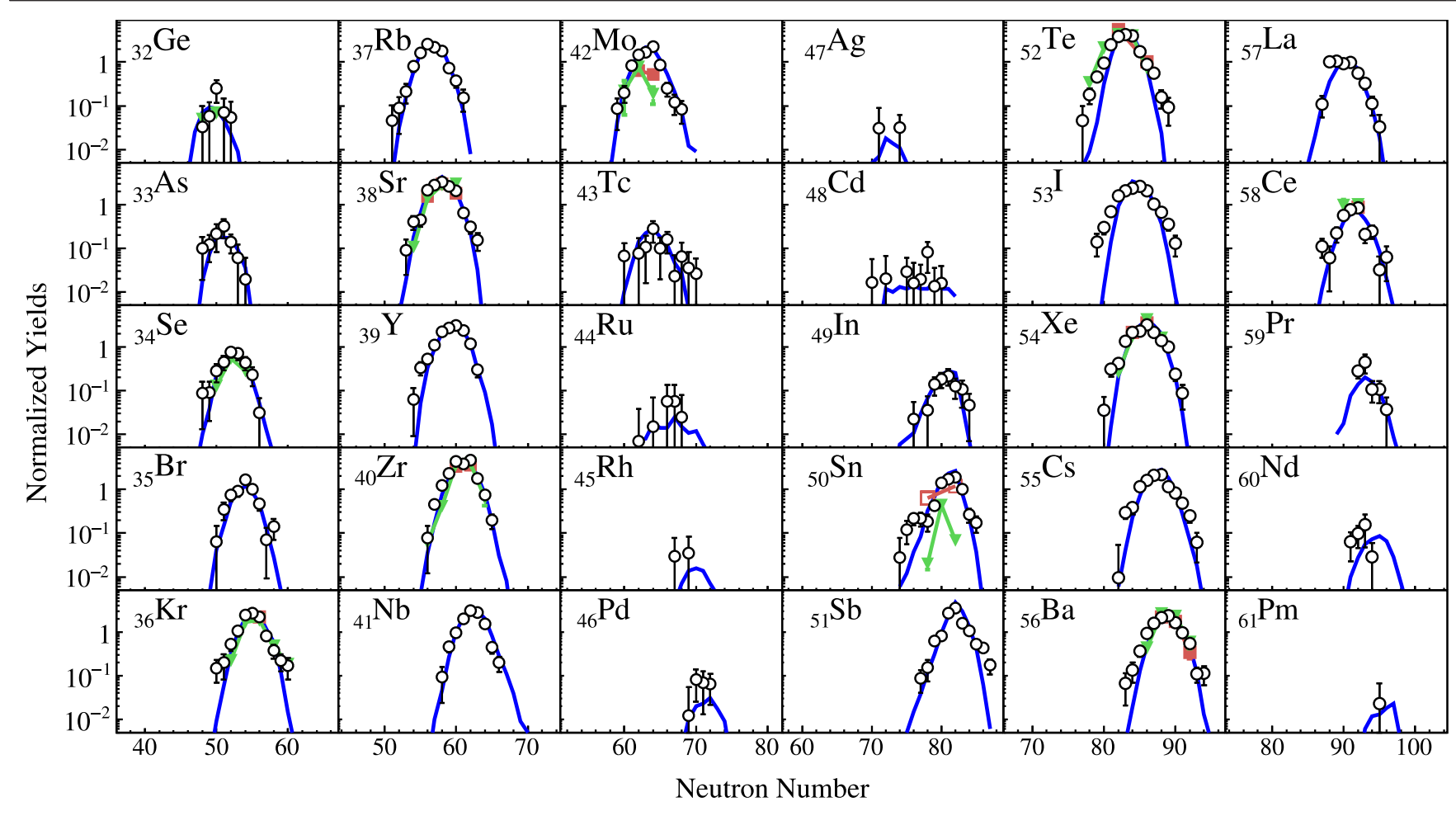

FIG. 2. Isotopic fission yields of ${ }^{239} \mathrm{U}$ (higher than $10^{-2}$ ): Each panel corresponds to an element, ranging from Ge to Pm. Yields are shown as functions of the neutron number. Present data (open circles) are compared with results of the GEF (v. 2018/1.1) [39] (blue lines) and with results of $\gamma$-spectroscopy measurements in Ref. [30] (green triangles and lines) and Ref. [40] (red squares and lines).

fragments from fusion-fission reactions was observed to be $(4 \pm 0.5) \%$ of the measured neutron-transfer-induced fission. This was isotopically subtracted, following the procedure described in Ref. [26]. This analysis procedure ensures that the contribution of other fissioning systems is lower than $0.9 \%$.

Isotopic-fission yields were derived, following the procedure presented in Refs. [23,26], within the range of excitation energy $0 \leq E_{x} \leq 15 \mathrm{MeV}$, resulting in a mean excitation energy of $8.3 \mathrm{MeV}$ with a standard deviation of 2.7 MeV. The total uncertainties presented in the data are obtained as the quadratic sum of statistical and systematic uncertainties. The systematic uncertainties, ranging from $2 \%$ in the heavier fragments up to $10 \%$ in the lighter ones, include those from the determination of the spectrometer acceptance, the relative normalization between both settings, and the contamination subtraction from fusionfission.

Isotopic fission yields of ${ }^{239} \mathrm{U}$ (open circles) are presented in Fig. 2 as a function of the fragment neutron number. These are compared with the semiempirical GEF model [39], which is commonly used in nuclear data evaluation. The GEF calculation (lines) was obtained using the measured excitation energy distribution and assuming a transferred angular momentum of $J=3 \hbar$ (the sensitivity to this parameter will be discussed later). The results of the $\gamma$-spectroscopy measurements of neutron-induced fission at $E_{x}=6.5 \mathrm{MeV}$ from Ref. [30] (green triangles) and Ref. [40] (red squares) are also shown. The yields for the intermediate elements are dominated by statistical fluctuations due to the strong asymmetry of the fission of ${ }^{239} \mathrm{U}$, with a very low production of $\mathrm{Rh}, \mathrm{Pd}$, and $\mathrm{Ag}$. A good agreement between the present data and the GEF calculations is obtained for the light fission products; whrease for the heavy fission products, the widths of the distributions are underestimated. A satisfactory agreement is also found between the present data and those obtained from $\gamma$ spectroscopy, except for Mo and $\mathrm{Sn}$ isotopes. In $\mathrm{Sn}$ isotopes, the data from Ref. [40] correspond to upper limits (unfilled red squares). The distributions of Mo and Sn, which are complementary isotopes, show mirror asymmetric shapes in both the present data and the GEF calculations. These shapes can be described in terms of different fission modes, as discussed in Ref. [25].

Fission yields of ${ }^{239} \mathrm{U}$ are shown in Fig. 3(a) as a function of the atomic number of the fission fragment. The present data (open circles) are compared with the GEF calculation (lines) and with the data obtained in the previous $\gamma$-spectroscopy measurements [30] (triangles). The present data show a strong even or odd effect that is fairly well reproduced by the GEF calculation. The asymmetric fission is also well reproduced by the GEF with an agreement within $10 \%$, except for $\mathrm{Te}$ and $\mathrm{Zr}$, which are underestimated. A good agreement is found between both 


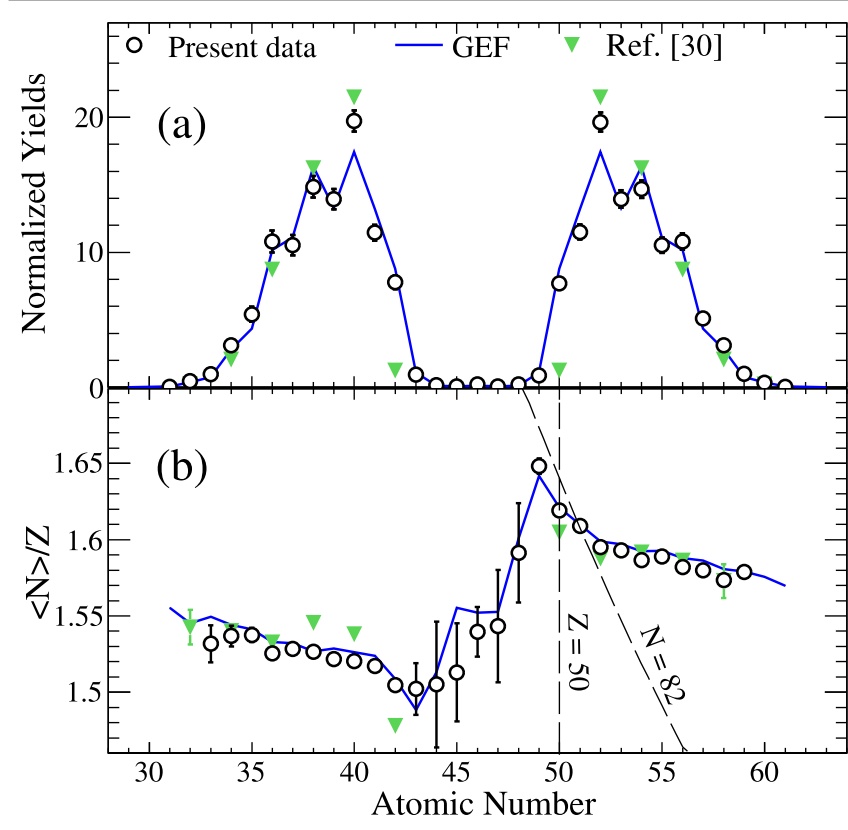

FIG. 3. Elemental fission yields and average neutron excess of fission fragments: (a) Normalized yield as a function of the atomic number. Present data (open circles) are compared with results of the GEF (v. 2018/1.1) [39] (blue line) and with data from $\gamma$ spectroscopy [30] (green triangles). (b) Average neutron excess of fission fragments as a function of atomic number. Spherical closed shells $Z=50$ and $N=82$ are indicated by dashed lines.

sets of experimental data for most of the elements within $\sim 20 \%$. However, large differences are observed in Mo and Sn, where the present dataset does not show the deviation by $600 \%$ with respect to the models reported in Ref. [30].

Figure 3(b) shows the average neutron excess for the fission fragments after neutron evaporation, which is defined as the average number of neutrons of each element divided by its atomic number, as a function of the atomic number. The dashed lines correspond to spherical closed shells $N=82$ and $Z=50$. The effect of these closed shells is clearly observed in the neutron excess of the heavy fragments, where the amount of neutrons of the fragments is locally enhanced due to the double magicity of ${ }^{132} \mathrm{Sn}$. The present data and the GEF calculations exhibit a very good agreement in the region of Sn. The data from $\gamma$ spectroscopy show significant fluctuations. Both Mo and Sn show a clear reduction of their mean neutron excess with respect to the present data. This is in contradiction with the expected increased neutron evaporation, which is mainly for the heavy fragment [41] due to the additional excitation energy of the present data $(+2 \mathrm{MeV})$. Consequently, the neutron excess should be reduced as compared to the $\gamma$-spectroscopy data. This opposite behavior suggests an experimental bias in the measured isotopic distribution from $\gamma$ spectroscopy for neutron-rich isotopes.

Figure 4 shows the evolution of the yield for Sn fragments from the fission of uranium as a function of the

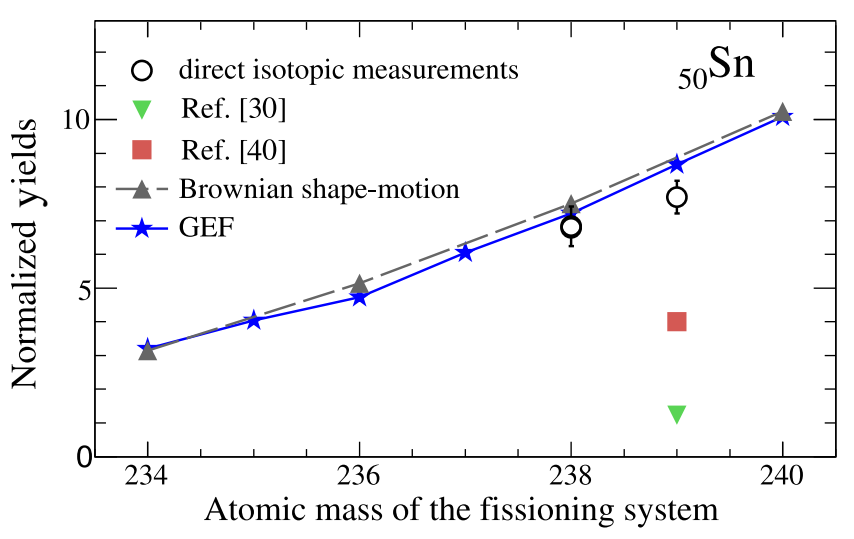

FIG. 4. Fission yields for $\mathrm{Sn}$ : Evolution of yields for $\mathrm{Sn}$ as a function of atomic mass of the fissioning system for the isotopic chain of uranium. Results of direct isotopic measurements obtained in this work and from Refs. [25,26] (open circles) are compared with a model based on the Brownian shape-motion, on 5D potential-energy surfaces [42] (dashed line), and with the GEF code (v. 2018/1.1) [39] (solid line). Data from $\gamma$-spectroscopy measurements of Ref. [30] (triangle) and Ref. [40] (square) are also included; in the last case, they are obtained from the complementary fragment.

atomic mass of the fissioning system. The Brownian shapemotion model with random walks on 5D potential-energy surfaces [42] predicts a continuous increase of the yield for $\mathrm{Sn}$ for heavier fissioning systems in the isotopic chain of uranium for initial excitation energy around $\sim 1 \mathrm{MeV}$ above the fission barrier. Similar values are also obtained using the GEF code. The data from both $\gamma$-spectroscopy measurements strongly disagree with these predictions, whereas the present data together with previous direct isotopic measurements for the fission of ${ }^{238} U[25,26]$ follow the trend and values predicted by the models. The large deviation of the fission yield for Sn obtained from $\gamma-\gamma$ coincidences [30] can be seen from Fig. 4. The fission yield obtained from single $\gamma$ spectroscopy [40] also has a large deviation, even if the reported value is closer to the present data and fission models.

The comparison performed in this work between the present data from neutron-transfer-induced fission and data from neutron-capture-induced fission might be affected by the different populations of angular momentum in both processes. Recent experimental results obtained from radiative neutron-capture processes and from surrogate reactions showed a strong enhancement of the $\gamma$-emission probability induced by the surrogate reaction with respect to the direct reaction [43], which was attributed to a larger angular momentum populated in the former reaction. However, this behavior was not observed in fission, where neutron-induced and transfer-induced fission showed similar probabilities [20] and fission-fragment distributions [26]. Small variations due to angular momentum were also predicted by theoretical models. The metropolis walk method combined with shape evolution, based on 
microscopically calculated level densities [44], predicts a negligible variation in yields of the atomic number for angular momenta above $J=2 \hbar$. The GEF code [39] estimates a variation lower than $5 \%$ in the yield of $\mathrm{Sn}$ for angular momenta in the range of $J=3-10 \hbar$. These observations, together with model predictions, exclude the influence of angular momentum in the discussion about the discrepancies in Mo and Sn between the present data and data from $\gamma$ spectroscopy [30,40].

The discrepancies between direct and $\gamma$-spectroscopy measurements may partially result from the bias arising from the $\gamma$-ray multiplicity experimental selection, as discussed in Ref. [40] and pointed out in the theoretical work of Ref. [45]. Furthermore, the $\gamma$-spectroscopy measurements rely on the precise knowledge of long-lived isomeric states that are known to occur in the Sn region (for instance, the $7^{-}$state with a half-life on the order of milliseconds in ${ }^{128} \mathrm{Sn}$ and ${ }^{130} \mathrm{Sn}$ [46]). All these transitions need to be considered to determine accurately the fission yields from $\gamma$-spectroscopy measurements. However, the knowledge of these transitions is far from being exhaustive today because new transitions are still being found $[47,48]$ and fission yields extracted using $\gamma$ spectroscopy could be underestimated. This further highlights the importance of direct and complete isotopic fission-fragment yields to obtain an accurate modeling of the fission process.

In summary, the first direct measurement of isotopic fission yields of ${ }^{239} \mathrm{U}$, performed using the neutron-transfer ${ }^{9} \mathrm{Be}\left({ }^{238} \mathrm{U},{ }^{239} \mathrm{U}\right){ }^{8} \mathrm{Be}$ reaction, is reported. An overall agreement on the fragment yields and $N / Z$ was achieved between the data and GEF calculations within 10\% accuracy. The increase of the production yield of $\mathrm{Sn}$ with respect to the fissioning mass was experimentally found in ${ }^{238} \mathrm{U}$ and ${ }^{239} \mathrm{U}$, which is in agreement with the predictions of the Brownian shape-motion model and the GEF. The present results disprove the fission yield anomaly for Mo and $\mathrm{Sn}$ isotopes reported from recent indirect $\gamma$-spectroscopy measurements. The survival of the asymmetric standard I fission mode at excitation energies of $2 \mathrm{MeV}$ above the fission barrier was confirmed for ${ }^{239} U$. This unique and complete dataset provides reliable constraints for fundamental fission models and valuable inputs for the evaluation of the heating inside of current reactors and the incineration capabilities of future reactors.

The authors acknowledge the excellent support from the GANIL technical staff, J. Goupil, G. Fremont, L. Menager, J. A. Ropert, and C. Spitaels. A. Navin is acknowledged for a careful reading of the Letter. D. R. thanks the PACS group (IPN Orsay) for the support and fruitful discussions. This work was partially supported by the Spanish Ministry of Research and Innovation under budget items FPA201571690-P and RYC-2012-11585, and by the Xunta de Galicia under item ED431F 2016/002. The research leading to these results has received funding from the European Union's HORIZON 2020 Program under Grant Agreement No. 654002 . *diego.ramos@ganil.fr

Present address: ILL, F-38042 Grenoble Cedex 9, France. †Present address: CEA, DAM, DIF, F-91297 Arpajon, France.

[1] L. Meitner and O. R. Frisch, Nature (London) 143, 239 (1939).

[2] O. Hahn and F. Strassmann, Naturwissenschaften 27, 11 (1939).

[3] 2018 OECD Nuclear Energy Agency Annual Report, Report No. 7416, 2018.

[4] Actinide and fission product partitioning and transmutation, OECD Nuclear Energy Agency Report No. 6996, 2012.

[5] J. Hamilton, A. Ramayya, S. Zhu, G. Ter-Akopian, Y. Oganessian, J. Cole, J. Rasmussen, and M. Stoyer, Prog. Part. Nucl. Phys. 35, 635 (1995).

[6] T. Wada, Y. Abe, and N. Carjan, Phys. Rev. Lett. 70, 3538 (1993).

[7] Y. A. Lazarev, I. I. Gontchar, and N. D. Mavlitov, Phys. Rev. Lett. 70, 1220 (1993).

[8] B. Jurado, C. Schmitt, K. H. Schmidt, J. Benlliure, T. Enqvist, A. R. Junghans, A. Kelić, and F. Rejmund, Phys. Rev. Lett. 93, 072501 (2004).

[9] P. Adrich, A. Klimkiewicz, M. Fallot, K. Boretzky, T. Aumann, D. Cortina-Gil, U. Datta Pramanik, T. W. Elze, H. Emling, H. Geissel, M. Hellström, K. L. Jones et al., Phys. Rev. Lett. 95, 132501 (2005).

[10] S. Goriely, J. L. Sida, J. F. Lemaître, S. Panebianco, N. Dubray, S. Hilaire, A. Bauswein, and H. T. Janka, Phys. Rev. Lett. 111, 242502 (2013).

[11] N. Schunck and L. M. Robledo, Rep. Prog. Phys. 79, 116301 (2016).

[12] K.-H. Schmidt and B. Jurado, Rep. Prog. Phys. 81, 106301 (2018).

[13] A. N. Andreyev, K. Nishio, and K.-H. Schmidt, Rep. Prog. Phys. 81, 016301 (2018).

[14] A. Bulgac, P. Magierski, K. J. Roche, and I. Stetcu, Phys. Rev. Lett. 116, 122504 (2016).

[15] G. Scamps and C. Simenel, Nature (London) 564, 382 (2018).

[16] B. D. Wilkins, E. P. Steinberg, and R. R. Chasman, Phys. Rev. C 14, 1832 (1976).

[17] U. Brosa, S. Grossmann, and A. Müller, Phys. Rep. 197, 167 (1990).

[18] P. Möller, D. G. Madland, A. J. Sierk, and A. Iwamoto, Nature (London) 409, 785 (2001).

[19] J. E. Escher, J. T. Burke, F. S. Dietrich, N. D. Scielzo, I. J. Thompson, and W. Younes, Rev. Mod. Phys. 84, 353 (2012).

[20] G. Kessedjian, B. Jurado, M. Aiche, G. Barreau, A. Bidaud, S. Czajkowski, D. Dassié, B. Haas, L. Mathieu, L. Audouin, N. Capellan, L. Tassan-Got et al., Phys. Lett. B 692, 297 (2010).

[21] K. Hirose, K. Nishio, S. Tanaka, R. Léguillon, H. Makii, I. Nishinaka, R. Orlandi, K. Tsukada, J. Smallcombe, M. J. Vermeulen, S. Chiba, Y. Aritomo et al., Phys. Rev. Lett. 119, 222501 (2017).

[22] K.-H. Schmidt, S. Steinhäuser, C. Böckstiegel, A. Grewe, A. Heinz, A. Junghans, J. Benlliure, H.-G. Clerc, M. de Jong, J. Müller, M. Pfützner, and B. Voss, Nucl. Phys. A665, 221 (2000). 
[23] M. Caamaño, O. Delaune, F. Farget, X. Derkx, K.-H. Schmidt, L. Audouin, C.-O. Bacri, G. Barreau, J. Benlliure, E. Casarejos, A. Chbihi, B. Fernández-Domínguez et al., Phys. Rev. C 88, 024605 (2013).

[24] J. L. Rodríguez-Sánchez, J. Benlliure, J. Taïeb, H. ÁlvarezPol, L. Audouin, Y. Ayyad, G. Bélier, G. Boutoux, E. Casarejos, A. Chatillon, D. Cortina-Gil, T. Gorbinet et al., Phys. Rev. C 91, 064616 (2015).

[25] E. Pellereau, J. Taïeb, A. Chatillon, H. Alvarez-Pol, L. Audouin, Y. Ayyad, G. Bélier, J. Benlliure, G. Boutoux, M. Caamaño, E. Casarejos, D. Cortina-Gil et al., Phys. Rev. C 95, 054603 (2017).

[26] D. Ramos, M. Caamaño, F. Farget, C. Rodríguez-Tajes, L. Audouin, J. Benlliure, E. Casarejos, E. Clement, D. Cortina, O. Delaune, X. Derkx, A. Dijon et al., Phys. Rev. C 97, 054612 (2018).

[27] M. A. Kellett, O. Besillon, and R. W. Mills, The JEFF-3.1/3.1.1 radioactive decay data and fission yields sub-libraries, OECD Nuclear Energy Agency Report No. 6287, 2009.

[28] D. Brown, M. Chadwick, R. Capote, A. Kahler, A. Trkov, M. Herman, A. Sonzogni, Y. Danon, A. Carlson, M. Dunn, D. Smith, G. Hale et al., ENDF/B-VIII, Nucl. Data Sheets 148, 1 (2018).

[29] Accelerator-driven systems (ADS) and fast reactors (FR) in advanced nuclear fuel cycles, OECD Nuclear Energy Agency Report No. 3109, 2002.

[30] J. N. Wilson, M. Lebois, L. Qi, P. Amador-Celdran, D. Bleuel, J. A. Briz, R. Carroll, W. Catford, H. De Witte, D. T. Doherty, R. Eloirdi, G. Georgiev et al., Phys. Rev. Lett. 118, 222501 (2017).

[31] D. J. and L. Hamilton, Nuclear Reactor Analysis (John Wiley \& Sons, New York, 1976).

[32] S. Marguet, The Physics of Nuclear Reactors (Springer, New York, 2017).

[33] S. Bjørnholm and J. E. Lynn, Rev. Mod. Phys. 52, 725 (1980).

[34] M. Rejmund, B. Lecornu, A. Navin, C. Schmitt, S. Damoy, O. Delaune, J. M. Enguerrand, G. Fremont, P. Gangnant, L. Gaudefroy, B. Jacquot, J. Pancin et al., Nucl. Instrum. Methods A 646, 184 (2011).
[35] C. Rodríguez-Tajes, F. Farget, X. Derkx, M. Caamańo, O. Delaune, K.-H. Schmidt, E. Clément, A. Dijon, A. Heinz, T. Roger, L. Audouin, J. Benlliure et al., Phys. Rev. C 89, 024614 (2014).

[36] Y. Kim, A. Lemasson, M. Rejmund, A. Navin, S. Biswas, C. Michelagnoli, I. Stefan, R. Banik, P. Bednarczyk, S. Bhattacharya, S. Bhattacharyya, E. Clément et al., Eur. Phys. J. A 53, 162 (2017).

[37] M. Vandebrouck, A. Lemasson, M. Rejmund, G. Fremont, J. Pancin, A. Navin, C. Michelagnoli, J. Goupil, C. Spitaels, and B. Jacquot, Nucl. Instrum. Methods A 812, 112 (2016).

[38] E. MacDonald, A. Shotter, D. Branford, J. Rahighi, T. Davinson, and N. Davis, Phys. Lett. B 283, 27 (1992).

[39] K.-H. Schmidt, B. Jurado, C. Amouroux, and C. Schmitt, Nucl. Data Sheets 131, 107 (2016).

[40] N. Fotiades, P. Casoli, P. Jaffke, M. Devlin, R. O. Nelson, T. Granier, P. Talou, and T. Ethvignot, Phys. Rev. C 99, 024606 (2019).

[41] A. A. Naqvi, F. Käppeler, F. Dickmann, and R. Müller, Phys. Rev. C 34, 218 (1986).

[42] P. Möller and C. Schmitt, Eur. Phys. J. A 53, 7 (2017).

[43] G. Boutoux, B. Jurado, V. Méot, O. Roig, L. Mathieu, M. Aïche, G. Barreau, N. Capellan, I. Companis, S. Czajkowski, K. H. Schmidt, J. T. Burke et al., Phys. Lett. B 712, 319 (2012).

[44] D. E. Ward, B. G. Carlsson, T. Døssing, P. Möller, J. Randrup, and S. Åberg, Phys. Rev. C 95, 024618 (2017).

[45] H. Paşca, A. V. Andreev, G. G. Adamian, N. V. Antonenko, and D. Lacroix, Phys. Rev. C 98, 014624 (2018).

[46] B. Fogelberg, K. Heyde, and J. Sau, Nucl. Phys. A352, 157 (1981).

[47] S. Pietri, A. Jungclaus, M. Górska, H. Grawe, M. Pfützner, L. Cáceres, P. Detistov, S. Lalkovski, V. Modamio, Z. Podolyák, P. H. Regan, D. Rudolph et al., Phys. Rev. C 83, 044328 (2011).

[48] G. S. Simpson, G. Gey, A. Jungclaus, J. Taprogge, S. Nishimura, K. Sieja, P. Doornenbal, G. Lorusso, P. A. Söderström, T. Sumikama, Z. Y. Xu, H. Baba et al., Phys. Rev. Lett. 113, 132502 (2014). 